(2) Open Access Full Text Article

\title{
An open-label, two-period comparative study on pharmacokinetics and safety of a combined ethinylestradiol/gestodene transdermal contraceptive patch
}

\author{
This article was published in the following Dove Press journal: \\ Drug Design, Development and Therapy \\ 10 March 2017 \\ Number of times this article has been viewed
}

\author{
Chao Zhang' \\ Haiyan $\mathrm{Li}^{2}$ \\ Xin Xiong' \\ Suodi Zhai \\ Yudong Wei ${ }^{2}$ \\ Shuang Zhang² \\ Yuanyuan Zhang' \\ Lin $X u^{2}$ \\ Li Liu' \\ 'Department of Pharmacy, ${ }^{2}$ Institute \\ of Clinical Trial, Peking University \\ Third Hospital, Beijing, People's \\ Republic of China
}

\begin{abstract}
We investigated the pharmacokinetics and safety profiles of a newly developed combined ethinylestradiol (EE)/gestodene (GSD) transdermal contraceptive patch after a single-dose administration and compared with the market available tablet formulation in healthy adult subjects. An open-label, two-period comparative study was conducted in 12 healthy women volunteers. A single dose of the study combined EE/GE transdermal contraceptive patch and oral tablet (Milunet ${ }^{\circledR}$ ) were administered. Blood samples at different time points after dose were collected, and concentrations were analyzed. A reliable, highly sensitive and accurate high-performance liquid chromatography coupled with tandem mass spectrometry (HPLC/MS/MS) assay method was developed in this study to determine the plasma concentrations of EE and GSD. Compared to the tablet, the study patch had a significantly decreased maximum plasma concentration $\left(C_{\max }\right)$, extended time to reach the $C_{\max }$ and half-life, as well as increased clearance and apparent volume of distribution. The half-lives of EE and GSD of the patch were 3.3 and 2.2 times, respectively, than the half-life of the tablet. The areas under the plasma concentration-time curve (AUCs) of EE and GSD of the patch were 8.0 and 16.2 times, respectively, than the AUC of the tablet. No severe adverse event was observed during the whole study, and the general safety was acceptable. In conclusion, compared to the oral tablet Milunet, the study contraceptive patch was well tolerated and showed potent drug exposure, significant extended half-life and stable drug concentrations.
\end{abstract}

Keywords: pharmacokinetics, safety, ethinylestradiol/gestodene, transdermal contraceptive patch

\section{Introduction}

Combined oral contraceptives (COCs) with estrogen and progestogen have been the most popular method of reversible birth control in most developed countries, and their use has increased dramatically in these areas. ${ }^{1}$ It is estimated that $>25 \%$ of all sexually active contracepting women in the US and 10\% in China rely on COCs. Concerns about using COCs focus on their safety and large fluctuations in serum hormone concentration. ${ }^{2-4}$ The risk of venous thromboembolism (VTE) and cardiovascular events is dose related with estrogen and progestogen. ${ }^{5}$ Meanwhile, it is recognized that poor compliance is common with daily COCs and can greatly reduce contraceptive efficacy. ${ }^{6,7}$ Therefore, over the years, the development of innovative, safe contraceptives associated with improved compliance has been important.
Correspondence: Chao Zhang Department of Pharmacy, Peking University Third Hospital, 49 Huayuan North Road, Haidian District, Beijing, People's Republic of China

Tel/fax +86 I0 82265740

Email laural.zhang@yahoo.com 
Challenges posted by the COCs may be directly addressed by a transdermal contraceptive delivery system (TCDS), which can provide continuous and stable serum levels of both estrogen and progestogen. Weekly administration may also greatly improve compliance compared to daily use of COCs, thereby reducing contraceptive failure rates. ${ }^{8,9}$ Moreover, TCDS avoids the loss of bioavailability because of first-pass hepatic metabolism and enzymatic degradation in the gastrointestinal tract, requiring lower drug doses to achieve similar efficacy. Accordingly, it can particularly reduce adverse effects associated with high plasma levels. ${ }^{10,11}$ The first transdermal contraceptive patch Ortho $\mathrm{Evra}^{\mathrm{TM}} / \mathrm{Evra}^{\mathrm{TM}}(0.75 \mathrm{mg}$ ethinylestradiol [EE] and $6 \mathrm{mg}$ norelgestromin [NGM] per patch; Janssen Pharmaceuticals, Inc., New Brunswick, NJ, USA) was approved by the US Food and Drug Administration (FDA) in 2001. However, different disadvantages were found subsequently, such as patch size limiting the amount that can be delivered, the system not suitable for high drug dose, only small and lipophilic drugs delivered through the skin and possible skin irritation and hypersensitivity reactions. Meanwhile, new formulation material and technology, such as a self-assembled nano-architecture liquid crystalline particles, have been developed as a promising TCDS carrier. ${ }^{12,13}$ Consequently, many new TCDSs containing more potent molecules have been developed, including transdermal patch made from Bayer Pharmaceuticals (Berlin, Germany) and a novel double-layer weekly sustained release transdermal patch..$^{14,15}$ Among newly developed progestogens, gestodene (GSD), a levonorgestrel derivative, offers the lowest available daily dose and exerts a more selective progestational action that improves cycle control, reducing metabolic changes and adverse effects while efficiently maintaining contraceptive efficacy. ${ }^{16,17}$ Therefore, the objectives of this study were to investigate the pharmacokinetics and safety of a newly developed combined EE/GSD transdermal contraceptive patch after single-dose administration and to compare it with that of the oral tablet formulation available in the market.

\section{Materials and methods Subjects}

It was intended that 12 healthy women aged $18-40$ years would be recruited and evaluated for pharmacokinetic analysis. The major inclusion criteria included body mass index (BMI) between 18 and 26; age difference $<10$ years; a healthy status on the basis of medical history, physical examination, laboratory tests and a willingness to refrain from consuming food or drink containing caffeine, xanthine, alcohol or grapefruit from $24 \mathrm{~h}$ before the administration of trial medication until the last blood sample for pharmacokinetic analysis had been obtained. Subjects had to smoke $<10$ cigarettes daily and be willing to refrain from smoking during the whole study. The major exclusion criteria included known or suspected pregnancy, contradictions to the use of the contraceptive patch or $\mathrm{COC}$, breastfeeding within 2 months before the trial, participation in other clinical trials within 3 months before the trial, use of contraceptives or any medication and substance that may influence the pharmacokinetic results determined by the investigator during the 4 weeks prior to the start of the trial, any acute or chronic systemic or local disease determined by the investigator, skin infection or damage that would influence the use of patch and positive hepatitis $\mathrm{B} / \mathrm{C}$ or HIV infection.

\section{Study design}

An open-label, two-period comparative study was conducted in a total of 12 healthy women volunteers. Two treatment periods comprised this study, and a single dose of the study combined EE/GSD transdermal contraceptive patch and the oral tablet formulation ( $30 \mu \mathrm{g}$ EE and $75 \mu \mathrm{g}$ GSD per tablet, Milunet $^{\circledR}$; Wyeth Pharma, S.A., Spain) were administered. Each transdermal patch was $20 \mathrm{~cm}^{2}$ (an active surface area of $10 \mathrm{~cm}^{2}$ ), containing $1.2 \mathrm{mg}$ EE and $1.6 \mathrm{mg}$ GSD. The multiple dosing regimen of the study patch was designed as three consecutive 7 -day patches (21 days) followed by 1 patch-free week per cycle. Therefore, in this study, a single dose of 7-day patch was investigated. Subjects were screened and hospitalized the night before the study started. After an overnight fast of at least $10 \mathrm{~h}$, each subject received a single oral dose of Milunet tablet with $240 \mathrm{~mL}$ of water on Day 1. Water intake was allowed from $2 \mathrm{~h}$ after dosing, and standardized meals were provided at 4 and $10 \mathrm{~h}$ after dosing. Blood samples were collected before and 0.25 , $0.5,0.75,1,1.5,2,3,4,6,8,10,12$ and $24 \mathrm{~h}$ after dosing through an indwelling venous catheter with anticoagulant. A 7-day washout duration was experienced, and all subjects were enrolled into hospital on Day 7 again for the second period. On Day 8, after overnight fasting for at least $10 \mathrm{~h}$, each subject received a single dose of the study contraceptive patch on her arm for 7 days (from Day 8 to Day 14). Blood samples were collected before and 0.25, 0.5, 0.75, 1 , $1.5,2,3,4,6,8,10,12,24,48,72,96,120,144,168,170$, $172,176,180,192,216$ and $240 \mathrm{~h}$ after dosing through an indwelling venous catheter with anticoagulant. All blood samples were centrifuged at $4^{\circ} \mathrm{C}$ at $2,500 \mathrm{rpm}$ for $10 \mathrm{~min}$. Plasma samples were stored at $-80^{\circ} \mathrm{C}$ until the assay. 
A posttreatment follow-up examination took place on Day 18 for all subjects. Adverse events (AEs) were observed and recorded during the whole study. Coffee, tea, chocolate, soda water, soft drinks, grapefruit and cruciferous vegetables were restricted for all subjects.

The study was approved by the China Food and Drug Administration (approval no 2012L01638) and Institute Ethical Review Board of Peking University Third Hospital. Written informed consent was obtained from all subjects prior to participation. The study was conducted in accordance with the Declaration of Helsinki 2013 and the laws and regulations of China.

\section{Drug concentration assay}

The plasma concentrations of EE and GSD were measured simultaneously using a validated high-performance liquid chromatography (Shimadzu LC-20A; Shimadzu MD, USA) coupled with tandem mass spectrometry (HPLC/MS/ MS; API 5500Qtrap; AB Sciex, Foster City, CA, USA) assay method, which we developed. A $500 \mu \mathrm{L}$ plasma sample was extracted by tert-butyl methyl ether first, and then, sulfonyl chloride acetone was used for derivatization. The lower limits of quantification (LLOQs) of EE and GSD were 10 and $200 \mathrm{pg} / \mathrm{mL}$, respectively, and their coefficients of variation were lower than $5.4 \%$ and $3.5 \%$, respectively. The calibration curves were linear over the concentration range of $10-1,000 \mathrm{pg} / \mathrm{mL}$ for $\mathrm{EE}$ and $200-20,000 \mathrm{pg} / \mathrm{mL}$ for GSD. The precisions of the assay for both EE and GSD were $<15 \%$, and recovery was $101.0 \%-105.8 \%$ and $82.1 \%-88.1 \%$ for $\mathrm{EE}$ and GSD, respectively. The samples under $0-10^{\circ} \mathrm{C}$ and room temperature were both stable for assay.

\section{Pharmacokinetic analysis}

The pharmacokinetic parameters of EE and GSD were calculated by non-compartmental analysis using Phoenix 64 WinNonlin 6.3 (Pharsight, Mountain View, CA, USA). The maximum plasma concentration $\left(C_{\max }\right)$ and the time to reach the $C_{\max }\left(\mathrm{t}_{\max }\right)$ were determined directly from the observed data. The area under the plasma concentration-time curve (AUC) from 0 to the last time $\left(\mathrm{AUC}_{0 \text {-last }}\right)$ was calculated using a linear trapezoidal rule. The terminal elimination rate constant $\left(\lambda_{z}\right)$ was estimated by log-linear regression analysis. The elimination half-life $\left(\mathrm{t}_{1 / 2}\right)$ and the apparent plasma clearance $(\mathrm{CL} / F)$ were calculated from the equations $\mathrm{t}_{1 / 2}=0.693 / \lambda_{Z}$ and $\mathrm{CL} / F$ $=$ dose/AUC, respectively. AUC from 0 to infinity $\left(\mathrm{AUC}_{0-\infty}\right)$ was calculated from the equation $\mathrm{AUC}_{0-\infty}=\mathrm{AUC}_{0-\text { last }}+C_{\mathrm{t}}$ $\lambda_{Z}$. The apparent volume of distribution $\left(V_{\mathrm{d}} / F\right)$ was obtained by the calculation from $C L / F$ and $\lambda_{Z}$.

\section{Safety assessment}

AEs were monitored throughout the study. All cohorts performed safety evaluations, including physical examinations, vital signs monitoring, 12-lead electrocardiogram (ECG), transvaginal and breast B ultrasound, cervix of the uterus thinprep cytology test inspection and laboratory tests, including hematology, serum chemistry and urinalysis. Systolic blood pressure (SBP), diastolic blood pressure (DBP) and pulse rate were measured in a sitting position before drug administration and at 1, 2, 5, 9, 169 and $176 \mathrm{~h}$ after dosing and during a follow-up visit. Twelve-lead ECG was taken at screening, prior to dosing of each period and during a follow-up visit. Laboratory tests were done pre and post dosing. Subjects were instructed to report all discomfort and AEs to the investigator, especially menstrual cycle and skin reactions.

\section{Results \\ Study participants}

A total of 12 healthy women were enrolled and completed the trial. Age ranged from 20 to 29 years, body weight ranged from 48.7 to $73.5 \mathrm{~kg}$, and BMI ranged from 18.8 to 25.7 (Table 1).

\section{Pharmacokinetics}

A total of 168 and 324 blood samples were taken after administration of tablet and the study patch, respectively. Considering shorter half-life of tablet compared to the study patch, blood samples were taken until $24 \mathrm{~h}$ after the tablet administration compared to $240 \mathrm{~h}$ for the study patch, of which most of their last time concentrations were below LLOQ. The mean plasma EE and GSD concentration versus time profiles after administration of a single dose of tablet and patch are shown in Figures 1 and 2, respectively. The pharmacokinetic parameters of EE and GSD for tablet and patch are shown in Table 2. Compared to the tablet, the contraceptive patch had a significantly decreased $C_{\max }$ and extended $\mathrm{t}_{\max }$ and half-life. The half-life of EE and GSD of the patch were 3.3 and 2.2 times that of the tablet, respectively. The AUCs of EE and GSD for the patch were 8 and 16.2 times that of the tablet, respectively. The comparisons of

Table I Demographic characteristics of subjects $(n=\mid 2)$

\begin{tabular}{llll}
\hline Characteristics & Mean & SD & Range \\
\hline Age (years) & 25 & 3 & $20-29$ \\
Body weight $(\mathrm{kg})$ & 58.4 & 6.8 & $48.7-73.5$ \\
Height $(\mathrm{cm})$ & 161 & 5 & $151-169$ \\
BMI $\left(\mathrm{kg} / \mathrm{m}^{2}\right)$ & 22.5 & 2.5 & $18.8-25.7$ \\
\hline
\end{tabular}

Abbreviations: SD, standard deviation; BMI, body mass index. 

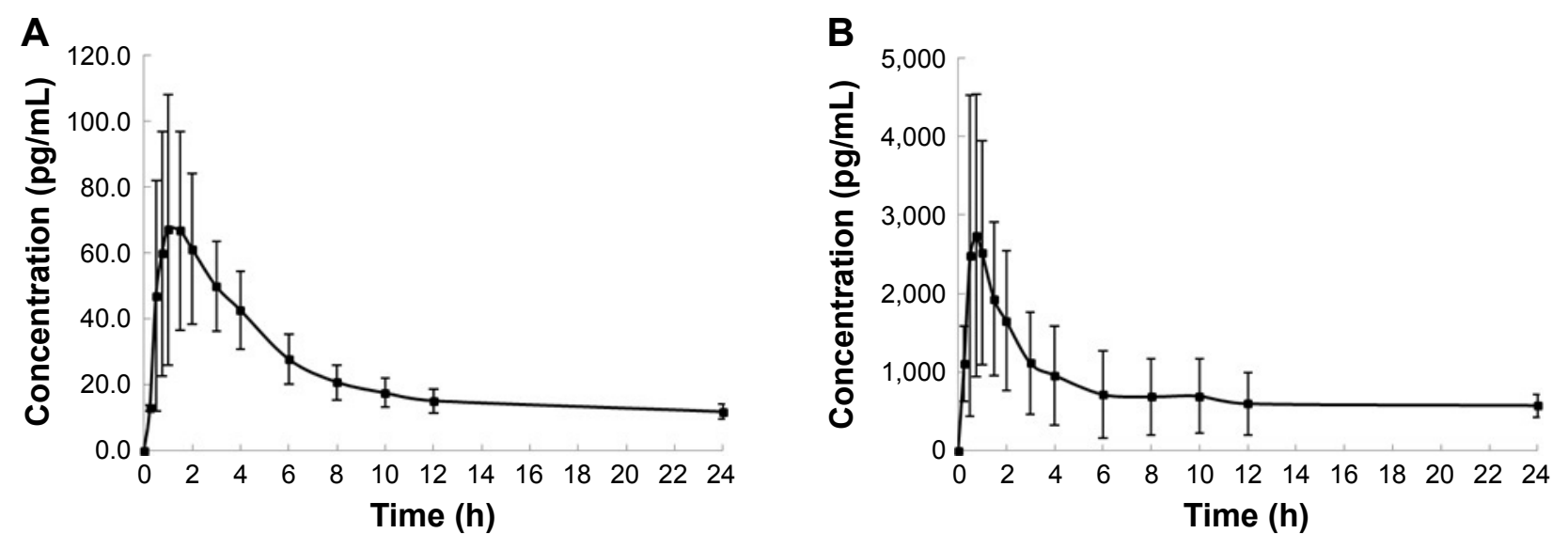

Figure I The mean plasma concentration versus time profiles of (A) EE and (B) GSD after a single-dose administration of tablet. Abbreviations: $\mathrm{EE}$, ethinylestradiol; GSD, gestodene.

main pharmacokinetic parameters of EE and GSD between the tablet and patch are listed in Table 3 .

\section{Safety}

No severe AEs were observed during the study. In total, there were five and 19 AEs found during the tablet and patch administration period, respectively (for tablet period: four cases of early menstruation and one case of dizziness and nausea; for patch period: eight cases of itch at the patching place, one case of reddish skin at the patching place, two cases of facial itch, three cases of neck acne with itch and five cases of menstruation disorders [three cases of early and two cases of postponed menstruation]). All AEs were of mild severity, and the subjects recovered without any sequelae or complications after no treatment. No black ring around worn patches and no clinically meaningful changes in physical examinations, blood pressure, heart rate and ECG were observed.

A

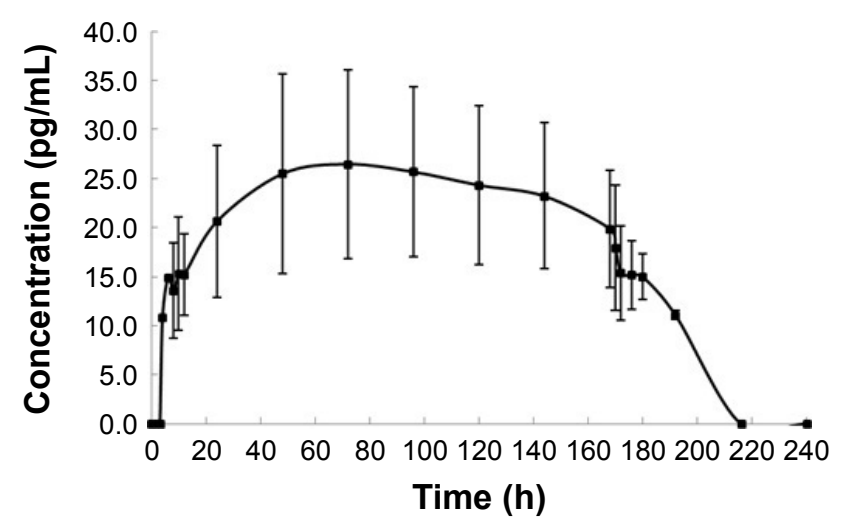

\section{Discussion}

This is the first study to investigate the pharmacokinetics and safety profiles of a newly developed combined EE/GSD transdermal contraceptive patch. Based on the results, the study contraceptive patch exhibits a potent drug exposure and is tolerable without any significant AEs. Compared to the commonly used oral tablet Milunet, the study contraceptive patch showed a decreased peak concentration, extended half-life and increased drug exposure, therefore exhibiting more stable and lasting effective concentrations.

TDES overcomes a number of disadvantages of COCs and is a promising formulation for contraceptive medications. TDES tenders sustaining administration of drug through the skin, which maintains stable plasma drug concentrations and avoids peaks and troughs. ${ }^{18}$ Reductions in bioavailability because of first-pass hepatic metabolism and enzymatic degradation in the gastrointestinal tract, which are seen with

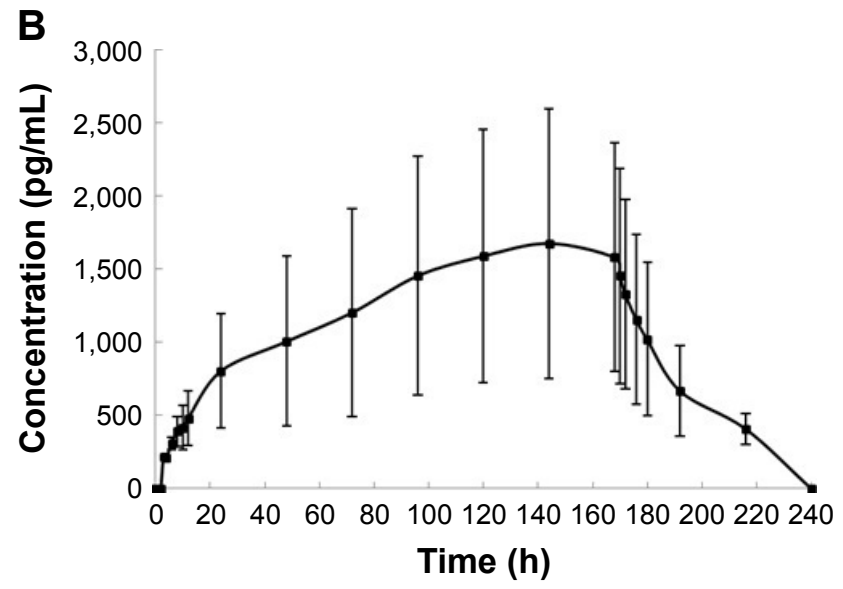

Figure 2 The mean plasma concentration versus time profiles of (A) EE and (B) GSD after a single-dose administration of the study patch. Abbreviations: EE, ethinylestradiol; GSD, gestodene. 
Table 2 The pharmacokinetic parameters of EE and GSD after a single-dose administration of $30 \mu \mathrm{g}$ EE/75 $\mu \mathrm{g}$ GSD tablet and 7 days wearing of $1.2 \mathrm{mg}$ EE/I.6 mg GSD study patch $(n=12)$

\begin{tabular}{|c|c|c|c|c|}
\hline \multirow[t]{2}{*}{ Parameters } & \multicolumn{2}{|c|}{ Tablet $($ mean \pm SD) } & \multicolumn{2}{|c|}{ Patch (mean \pm SD) } \\
\hline & EE & GSD & EE & GSD \\
\hline$C_{\max }(\mathrm{pg} / \mathrm{mL})$ & $74.1 \pm 35.6$ & $3,060 \pm 1,976$ & $28.8 \pm 10.3$ & $\mathrm{I}, 720 \pm 894$ \\
\hline$t_{\max }(h)$ & $1.5 \pm 0.5$ & $0.9 \pm 0.4$ & $86 \pm 31$ & $144 \pm 29$ \\
\hline$t_{1 / 2}(h)$ & $8.4 \pm 4.8$ & $9.6 \pm 5.8$ & $27.7 \pm 34.2$ & $21.1 \pm 4.7$ \\
\hline $\mathrm{CL} / F(\mathrm{~L} / \mathrm{h})$ & $58.0 \pm 19.8$ & $7.9 \pm 6.6$ & $303.5 \pm 100.5$ & $8.5 \pm 4.9$ \\
\hline$V_{\mathrm{d}} / F(\mathrm{~L})$ & $625.3 \pm 228.7$ & $65.1 \pm 26.6$ & 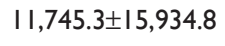 & $255.4 \pm \mid 35.6$ \\
\hline $\mathrm{AUC}_{0 \text {-last }}(\mathrm{pg} \cdot \mathrm{h} / \mathrm{mL})$ & $432.0 \pm 183.9$ & $14,2 \mid 8 \pm 12,290$ & $3,850 \pm I, 388$ & $238,119 \pm 127,415$ \\
\hline$A \cup C_{0-\infty}(p g \cdot h / m L)$ & $487.4 \pm 166.6$ & $14,976 \pm 1 \mid, 905$ & $3,895 \pm I, 423$ & $242,844 \pm 128,196$ \\
\hline
\end{tabular}

Abbreviations: $C_{\max }$, maximum plasma concentration; $\mathrm{t}_{\max }$, time to reach the $C_{\max } ; \mathrm{t}_{1 / 2}$, elimination half-life; CL/F, apparent plasma clearance; $V_{\mathrm{d}} / F$, apparent volume of distribution; $A \cup C_{0-\text { last }}$, area under the plasma concentration-time curve from 0 to the last time; $A \cup C_{0-\infty}$, area under the plasma concentration-time curve from 0 to infinity; $\mathrm{EE}$, ethinylestradiol; GSD, gestodene; SD, standard deviation.

oral drug administration, are avoided.$^{19}$ The multi-day dosing would improve poor patient compliance due to frequent dosing when given orally. Adherence has been shown to be directly related to contraceptive outcomes. ${ }^{9}$ Contraceptive failure among women using COCs is primarily due to poor compliance. TDES may reduce systemic side effects, particularly those that are linked to high plasma concentrations. Extra advantages of TDES have involved an alternative administration route for patients who are unable to take oral medications and the immediate cessation of drug administration with removal of the patch. ${ }^{9}$

Compared to the TCDS on the market (Ortho Evra ${ }^{\mathrm{TM}} /$ Evra $^{\mathrm{TM}}$, containing $0.75 \mathrm{mg}$ EE and $6 \mathrm{mg}$ NGM), the study contraceptive patch chose a levonorgestrel derivative GSD as the component. NGM is the primary active metabolite of norgestimate and is also partially converted to the more potent progestin levonorgestrel. ${ }^{20}$ Therefore, GSD offers low daily dosing with advantages of a more selective action, reducing metabolic changes and adverse effects. Also, EE is the most potent estrogen agonist currently available and both EE and GSD are well absorbed through the skin. ${ }^{3}$ Each study patch contains1.2 mg EE and $1.6 \mathrm{mg}$ GSD,

Table 3 The comparisons of main pharmacokinetic parameters of EE and GSD between a single-dose administration of $30 \mu \mathrm{g}$ $\mathrm{EE} / 75 \mu \mathrm{g}$ GSD tablet and 7 days wearing of $1.2 \mathrm{mg} \mathrm{EE} / \mathrm{l} .6 \mathrm{mg}$ GSD study patch

\begin{tabular}{lll}
\hline Parameters & \multicolumn{2}{l}{ Patch:tablet ratio (mean value) } \\
\cline { 2 - 3 } & EE & GSD \\
\hline$C_{\max }$ & 0.4 & 0.6 \\
$\mathrm{t}_{\max }$ & 57.3 & 160.0 \\
$\mathrm{t}_{1 / 2}$ & 3.3 & 2.2 \\
AUC $_{0-\infty}$ & 8.0 & 16.2 \\
\hline
\end{tabular}

Abbreviations: $C_{\max }$, maximum plasma concentration; $\mathrm{t}_{\max }$, time to reach the $C_{\max }$; $\mathrm{t}_{1 / 2}$, elimination half-life; $A \cup \mathrm{C}_{0-\infty}$, area under the plasma concentration-time curve from 0 to infinity; EE, ethinylestradiol; GSD, gestodene. which are comparable with the components of Bayer patch involving $0.55 \mathrm{mg} \mathrm{EE}$ and $2.1 \mathrm{mg}$ GSD per patch (Bayer HealthCare AG, Berlin, Germany). For Bayer patch, Hofmann et $\mathrm{al}^{21}$ reported that the half-lives and $\mathrm{AUC}_{0-168}$ of $\mathrm{EE}$ and GSD were 22.9 and $27.1 \mathrm{~h}$ and 4,533 and $63,700 \mathrm{pg} \cdot \mathrm{h} /$ $\mathrm{mL}$, respectively. Meanwhile, $\mathrm{AUC}_{0-168}$ values of $\mathrm{EE}$ and GSD were investigated by Westhoff et $\mathrm{al}^{22}$ as 3,527 and $598,000 \mathrm{pg} \cdot \mathrm{h} / \mathrm{mL}$, respectively, and the clearance of EE was found to be increased with body weight within the range of their study (48.3-166 kg), but limited effect on GSD. Comparing the study patch with the Bayer patch, the half-life and AUC of EE had no major differences, but AUC of GSD of the study patch was much smaller than that of the Bayer patch. These differences may be related to the relative low body weight of the subjects included in this study ( $58.4 \mathrm{~kg}$ of average body weight; Table 1), lower dose of GSD, different treatment periods, decreased skin absorption, etc.

The mean $C_{\max }$ values of EE and GSD after administration of a single dose of the study transdermal patch were found to be only $40 \%$ and $60 \%$ of those of the oral tablet, which may be related to the decreased adverse drug effect occurrences. The half-lives of both EE and GSD were observed to be significantly extended approximately two to three times compared to those after oral tablet administration. Blood sample interval inconsistence (until $24 \mathrm{~h}$ for oral tablet and $240 \mathrm{~h}$ for the study patch) may account for part of half-life differences. Figure 2 shows slow absorption and lasting concentrations of both EE and GSD after administration of patch, but relative increase in clearances. Therefore, the study patch behaved a constant, long-lasting, less fluctuant concentration, which contributed to maintaining contraceptive efficacy. Meanwhile, AUC values of EE and GSD demonstrated that drug exposures of the study patch were more potent than those of tablet, 8.0 and 16.2 times for EE 
and GSD, respectively, compared with oral tablet. Different treatment periods, single application for tablet and 7 days patching for the study patch, would potentially influence the AUC calculation.

Moreover, GSD had a high affinity binding to sex hormone binding globulin (SHBG). Therefore, the free drug concentration and distribution of GSD were known to be influenced by SHBG. ${ }^{23}$ Furthermore, it was found that COCs containing desogestrel or GSD cause an average SHBG increase of $200 \%-300 \%$, which further complicates the disposition of GSD. ${ }^{24}$ Accordingly, it was reported that the concentrations of GSD during multiple dosing could not be predicted on the basis of single-dose pharmacokinetics. ${ }^{25}$ SHBG was not determined in this study, and therefore, further studies on SHBG potential effects and pharmacokinetics of multiple dose of the study patch should be developed.

The study EE/GSD transdermal patch had an active surface area of $10 \mathrm{~cm}^{2}$, and the daily absorption from vitro study results was $20 \mu \mathrm{g}$ for EE and $60 \mu \mathrm{g}$ for GSD, respectively. The total patch area was $20 \mathrm{~cm}^{2}$, which was surrounded by a perimeter adhesive system to improve skin adhesion. Therefore, it also had potential compliance-related advantages because of the ease and simplicity of use.

Administration of the study transdermal patch was well tolerated. Menstruation disorders were common AEs after administration of both patch and oral tablet. It is reasonably understood that estrogen and progestogen influence the menstruation period and induce early or postponed menstruation, which is usually seen in all contraceptives. ${ }^{26}$ The most common AE was skin itch or redness at the patch placement site, which was observed in nine subjects out of a total of 12 subjects in this study. It was thought to be related to the matrix materials, and therefore, the biocompatibility may need to be further improved to reduce the local irritation. All AEs were of mild severity and recovered without any sequelae or complications after no treatment. No black ring around worn patches and bleeding were observed, which may be due to short treatment period investigated in this study.

In this study, the pharmacokinetics and safety of a singledose administration of the study patch were investigated. The characteristics of multiple doses should be further evaluated in future studies. Moreover, it was reported that the transdermal contraceptive patch may be less effective in women with body weight $>90 \mathrm{~kg} .{ }^{26}$ Therefore, investigating this special population is also valuable. Small sample size and no evaluation of SHBG potential influence were limitations of this study. Although subjects included in this study were required to synchronize with their menstrual cycles, omissions may could not be totally avoided.

\section{Conclusion}

Single-dose administration of the study EE/GSD contraceptive patch to healthy individuals was well tolerated with no severe AEs. Compared to the oral tablet Milunet, the study contraceptive patch showed a potent drug exposure and significant extended half-life for both EE and GSD, indicating a longer, stable and potent contraceptive efficacy.

\section{Acknowledgment}

The authors thank Lisa Chen for reviewing the language in the manuscript.

\section{Disclosure}

The authors report no conflicts of interest in this work.

\section{References}

1. Daniels K, Daugherry J, Jones J. Current contraceptive status among women aged 15-44: United States, 2011-2013. NCHS Data Brief. 2014;(173):1-8.

2. Rosenberg MJ, Waugh MS, Meehan TE. Use and misuse of oral contraceptives: risk indicators for poor pill taking and discontinuation. Contraception. 1995;51(5):283-288.

3. Kuhl H. Pharmacology of estrogens and progestogens: influence of different routes of administration. Climacteric. 2005;8(suppl 1):3-63.

4. Jung Hoffmann $\mathrm{C}$, Kuhl H. Intra- and interindividual variations in contraceptive steroid levels during 12 treatment cycles: no relation to irregular bleedings. Contraception. 1990;42(4):423-438.

5. Sidney S, Cheetham TC, Connell FA, et al. Recent combined hormonal contraceptives (CHSs) and the risk of thromboembolism and other cardiovascular events in new users. Contraception. 2013;87(1):93-100.

6. Rosenberg MJ, Waugh MS. Oral contraceptive discontinuation: a prospective evaluation of frequency and reasons. Am JObstet Gynecol. 1998;179(3 pt 1):577-582.

7. Aubeny E, Buhler M, Colau JC, Vicaut E, Zadikian M, Childs M. The Coraliance study: non-compliant behavior. Results after a 6-month follow-up of patients on oral contraceptives. Eur J Contracept Reprod Health Care. 2004;9(4):267-277.

8. Potts RO, Lobo RA. Transdermal drug delivery: clinical considerations for the obstetrician-gynecologist. Obstet Gynecol. 2005;105(5 pt 1): 953-961.

9. Burkman RT. Transdermal hormonal contraception: benefits and risks. Am J Obstet Gynecol. 2007;197(2):134.e1-134.e6.

10. Samisoe G. Transdermal hormone therapy: gels and patches. Climacteric. 2004;7(4):347-356.

11. Van den Heuvel MW, van Bragt AJ, Alnabawy AK, et al. Comparison of ethinylestradiol pharmacokinetics in three hormonal contraceptive formulations: the vaginal ring, the transdermal patch and an oral contraceptive. Contraception. 2005;72(3):1646-1652.

12. Elgindy NA, Mehanna MM, Mohyeldin SM. Self-assembled nano-architecture liquid crystalline particles as a promising carrier for progesterone transdermal delivery. Int J Pharm. 2016;501(1-2): $167-179$.

13. Mohyeldin SM, Mehanna MM, Elgindy NA. Superiority of liquid crystalline cubic nanocarriers as hormonal transdermal vehicle: comparative human skin permeation-supported evidence. Expert Opin Drug Deliv. 2016;13(8):1049-1064. 
14. Gruber D, Skřivánek A, Serrani M, Lanius V, Merz M. A comparison of bleeding patterns and cycle control using two transdermal contraceptive systems: a multicenter, open-label, randomized study. Contraception. 2015;91(2):105-112.

15. Gao Y, Liang J, Liu J, Xiao Y. Double-layer weekly sustained release transdermal patch containing gestodene and ethinylestradiol. Int $J$ Pharm. 2009;377(1-2):128-134.

16. Wilde MI, Balfour JA. Gestodene. A review of its pharmacology, efficacy and tolerability in combined contraceptive preparations. Drugs. 1995;50(2):364-395.

17. Barbosa IC, Filho CI, Faggion D Jr, Baracat EC. Prospective, open-label, noncomparative study to assess cycle control, safety and acceptability of a new oral contraceptive containing gestodene 60 microg and ethinylestradiol 15 microg (Minesse). Contraception. 2006;73(1):30-33.

18. Nelson AL. Transdermal contraception methods: today's patches and new options on the horizon. Expert Opin Pharmacother. 2015;16(6): 863-873.

19. Abrams LS, Skee D, Natarajan J, Wong FA. Pharmacokinetic overview of Ortho Evra'TM/Evra ${ }^{\mathrm{TM}}$. Fertil Steril. 2002;77(2 suppl 2):S3-S12.

20. Sriprasert I, Stanczyk FZ, Archer DF. Ethinyl estradiol and levonorgestrel in a transdermal contraceptive delivery system. Expert Opin Pharmacother. 2015;16(12):1901-1909.

21. Hofmann B, Reinecke I, Schuett B, Merz M, Zurth C. Pharmacokinetic overview of ethinyl estradiol dose and bioavailability using two transdermal contraceptive systems and a standard combined oral contraceptive. Int J Clin Pharmacol Ther. 2014;52(12):1059-1070.
22. Westhoff CL, Reinecke I, Bangerter K, Merz M. Impact of body mass index on suppression of follicular development and ovulation using a transdermal patch containing 0.55-mg ethinyl estradiol/2.1-mg gestodene: a multicenter, open-label, uncontrolled study over three treatment cycles. Contraception. 2014;90(3):272-279.

23. Bergink W, Assendorp R, Kloosterboer L, van Lier W, Voortman G, Qvist I. Serum pharmacokinetics of orally administered desogestrel and binding of contraceptive progestogens to sex hormone-binding globulin. Am J Obstet Gynecol. 1990;163(6 pt 2):2132-2137.

24. Odlind V, Milsom I, Persson I, Victor A. Can changes in sex hormone binding globulin predict the risk of venous thromboembolism with combined oral contraceptive pills? Acta Obstet Gynecol Scand. 2002; 81(6):482-490.

25. Back DJ, Power J, Winkler U, et al. Pharmacokinetics and protein binding of 3-ketodesogestrel and gestodene in the serum of women during 6 cycles of treatment with two low dose oral contraceptives. Adv Contracept. 1993;9(4):319-330.

26. Zieman M, Guillebaud J, Weisberg E, Shangold GA, Fisher AC, Creasy GW. Contraceptive efficacy and cycle control with the Ortho Evra $^{\mathrm{TM}} / \mathrm{Evra}^{\mathrm{TM}}$ transdermal system: the analysis of pooled data. Fertil Steril. 2002;77(2 suppl 2):S13-S18.
Drug Design, Development and Therapy

\section{Publish your work in this journal}

Drug Design, Development and Therapy is an international, peerreviewed open-access journal that spans the spectrum of drug design and development through to clinical applications. Clinical outcomes, patient safety, and programs for the development and effective, safe, and sustained use of medicines are the features of the journal, which

\section{Dovepress}

has also been accepted for indexing on PubMed Central. The manuscript management system is completely online and includes a very quick and fair peer-review system, which is all easy to use. Visit http://www.dovepress.com/testimonials.php to read real quotes from published authors.

Submit your manuscript here: http://www.dovepress.com/drug-design-development-and-therapy-journal 